\title{
The temporal endoscopic midface lift - centrofacial rejuvenation without facial scars
}

\author{
Thomas Haffner \\ Heumarkt Clinic Cologne, 50667 Koeln, Germany.
}

Correspondence to: Dr. Thomas Haffner, Heumarkt Clinic Cologne, Heumarkt 43, 50667 Koeln, Germany. E-mail: praxishaffner@gmail.com

How to cite this article: Haffner T. The temporal endoscopic midface lift - centrofacial rejuvenation without facial scars. Plast Aesthet Res 2016;3:339-46.

Article history:

Received: 05-08-2016

Accepted: 17-10-2016

Published: 31-10-2016

Key words:

Facelift,

midface,

endoscopic facelift,

temporal endoscopic midface lifting,

facial scar,

centrofacial,

rejuvenation

\begin{abstract}
Aim: The author describes a new endoscopic midface lifting technique using solely temporal access and evaluates its advantages and perspectives. Methods: This is a single-surgeon case study. Through a short temporal incision, dissection is performed along a single plane connecting the superficial surfaces of both the facial and temporal superficial musculoaponeurotic system (SMAS) layers. Subsequent midface dissection divides the malar fat pad into the deeper and superficial parts. A new concept of a combination high malar SMAS lift and internal skin flap anchorage that provides long-term stability is described. Results: The temporal endoscopic midface (TEM) lift technique resulted in good objective results with high patient satisfaction. The temporal access allowed proper vertical vector correction of the sagging centro-facial structures. The resulting scar was inconspicuous and hidden in the hair bearing skin, and its length was measured between 5-6 $\mathrm{cm}$. A conversion to the conventional long facial scar approach was completely avoided. Conclusion: The TEM lift is a new and effective procedure for facial rejuvenation, especially the midface and cheek. The results illustrate the importance of internal anchorage of both the midface SMAS and skin flap. These are the keys to longlasting and pleasing results. The procedure is best suited for younger men and women with little or no neck skin laxity.
\end{abstract}

\section{INTRODUCTION}

The midface is of central importance in facial aging as this is the first site where its signs manifest themselves. These signs comprise volume loss, deflation, malar prominence flattening, baggy eyes and development of nasolabial and nasojugal folds due to ptosis and laxity. The midface lift reverses the ptosis by reposition of the sagging tissues and has even been dubbed the the facelift of the 21 st century by Botti and Ceravolo ${ }^{[1]}$ due to its effectiveness. The midface lift has been performed using the transblepharoplasty, ${ }^{[1,2]}$ trans-oral, ${ }^{[3]}$ trans-temporal ${ }^{[4-8]}$ or brow-lift ${ }^{[9]}$ approach. The trans-blepharoplasty approach has all the associated disadvantages of lower lid surgery including a canthopexy requirement and risks of asymmetry, scleral show, lagophtalmus and ectropion development. With the trans-temporal subperiosteal midface lift, dissection is extensive, technically demanding, has greater risks and a

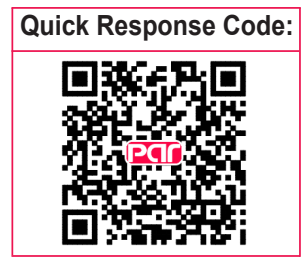


longer recovery. The dissection plane has to be developed under the superficial temporal fascia and then transitioned to the subperiosteal plane over the zygoma. The surfaces of the zygoma and malar bone then need to be connected through subperiosteal dissection. Subsequent anchorage of the malar tissues are achieved only by suspension of the malar fat pad ${ }^{[8]}$ and its longevity is questionable. Other alternative procedures such as the minimal access cranial suspension (MACS) lift with a third suture ${ }^{[10]}$ cause a visible facial scar of $14-16 \mathrm{~cm}$ in length.

\section{METHODS}

The temporal endoscopic midface (TEM) lift is a new minimal-access facelift that uses exclusively temporal access incisions thereby sparing any scars on the face itself. This utilizes endoscopic dissection and a suturing technique that was developed by the author. ${ }^{[11]}$ An incision measuring $5-6 \mathrm{~cm}$ is made and hidden in the hair-bearing part of the temple. Common pitfalls such as damaging the hair roots or making the flap excessively thin must be avoided at this stage. The dissection plane is developed over the superficial surface of the common facial and temporal superficial musculoaponeurotic system (SMAS). It is important to completely avoid violating the integrity of the SMAS by either inadvertent incisions or diathermy.

An alternative initial approach through the same incision is a dual plane dissection [Figure 1]. In this approach, the plane between the superficial and deep layers of the temporal fascia is first developed and dissected towards the non-hair bearing skin of the face. When or before the junction of the hairline is reached, an incision is made on the deep surface of the

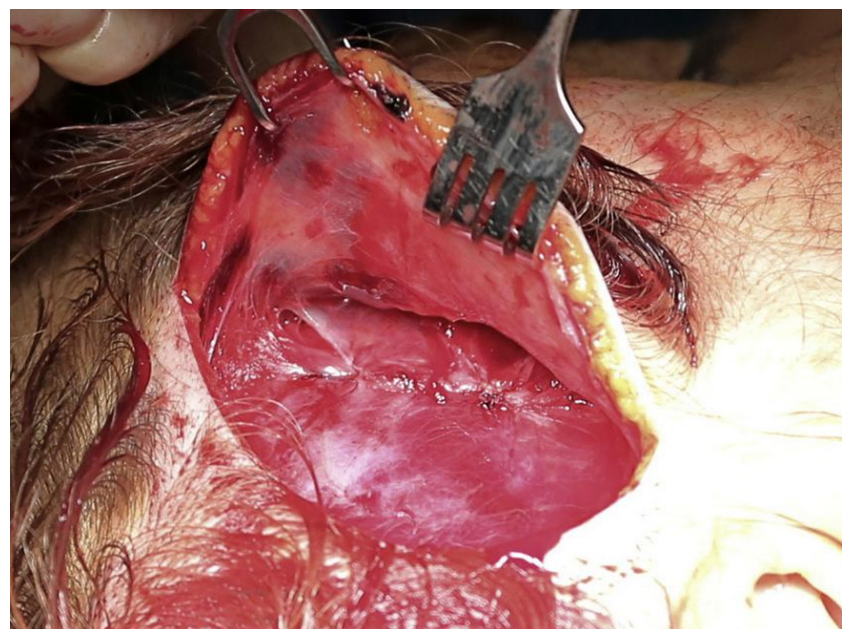

Figure 1: Dual plane temporal dissection. The superficial plane above the superficial fascia is exposed after dissecting the deep plane first. The superficial fascia is fixed by sutures onto the deeper one in order to facilitate the dissection superficial layer of the temporal fascia. The dissecting plane is then transitioned onto the superficial surface of the superficial layer of the temporal fascia.

The subsequent surgical steps are common to both approaches. As dissection proceeds over the zygoma, the temporal branch of the facial nerve remains protected, it is deep to the superficial layer of the temporal fascia. Both the mid-facial (malar) SMAS and the lateral facial SMAS can be easily reached this way.

\section{Pertinent anatomy}

The temporal and facial portions of the SMAS fuse over the malar bone. The facial SMAS continues in the temple as the superficial temporal fascia ${ }^{[1]}$ and the frontal branch of the facial nerve lies deep to it. Limiting dissection to the plane above this fascia without violating it will ensure avoidance of nerve injury. ${ }^{[1]}$ The facial SMAS continues in the midface as the malar SMAS and is incorporated by the thick malar fat pad.

The anatomy of the facial fat compartments and that of the malar fat pad was best described by Botti and Ceravolo ${ }^{[1]}$ through cadaveric studies. The malar fat pad was found to be divided into two parts, a superficial part and a deeper part.

The author's clinical experience is consistent with these findings. The midface can be well visualised through a medial extended facelift [Figure 2] and the parts of the malar fat pad can be easily distinguished. The superficial part originates from the skin and can be conceptualised as a condensation of the malar thickening of skin fat with the strong Camper's fascia. Under this, the deep part of the malar fat pad is found

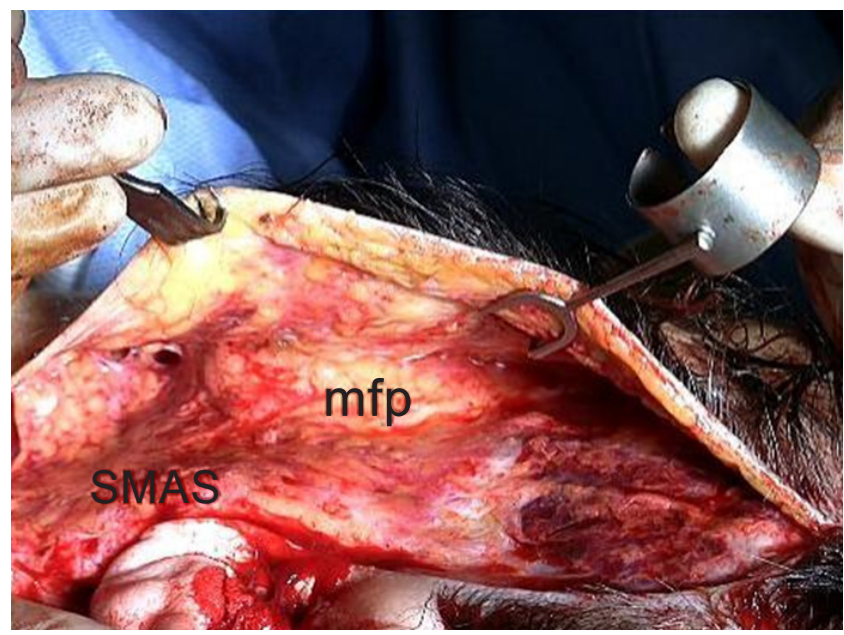

Figure 2: Demonstration of the midface with the malar fat pad (malar SMAS) during an extended temporal-cervical-facial open facelift. SMAS: superficial musculoaponeurosis system; mfp: malar fat pad 


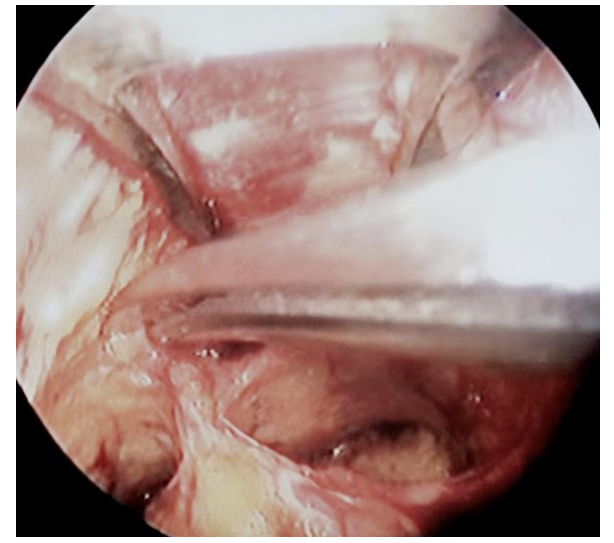

Figure 3: Mobilising of the midface by endoscopic dissection of the retaining ligaments

enmeshed and reinforced by the fibres of the SMAS. Both the superficial and deep parts of the fat pad hold sutures well as the intertwined Camper's fascia and SMAS fibres respectively, lend them strength. This unique anatomical construct of the reinforced malar fat pad serves as an effective pole for which lifting and anchoring threads can be secured to with effectiveness and longevity.

The key elements of the method are the endoscopic dissection in the superficial plane, the high malar SMAS anchor, the direct internal flap anchor and the author's suture technique in the tunnel and keyhole access.

\section{Endoscopic dissection}

Once the plane of dissection has progressed beyond the zygoma, a rigid $4 \mathrm{~mm}$ diameter operative endoscope with a 30-degree angle is used to visualise this area for dissection. The endoscopic dissector is introduced over the endoscope through the temporal incision. A distal spoon-shaped shield is also used to maintain proper visualization of the optical cavity.

A hollow space is created using the endocopic dissector to progressively elevate the skin. The internal facial structures are visible throughout the dissection. The endpoint for a good working cavity is reached after release of the fasciocutaneous ligaments, especially the zygomatic and the parotidomasseteric ligaments. Attention must be paid to avoid incorrectly identifying ligaments that are branches of the facial nerves [Figure 3].

SMAS excision is not mandatory as good results can already be achieved in conventional facelifts without it. The most challenging element of the procedure is maintaining dissection in the correct plane. Adherence to this avoids any damage to the facial nerve.

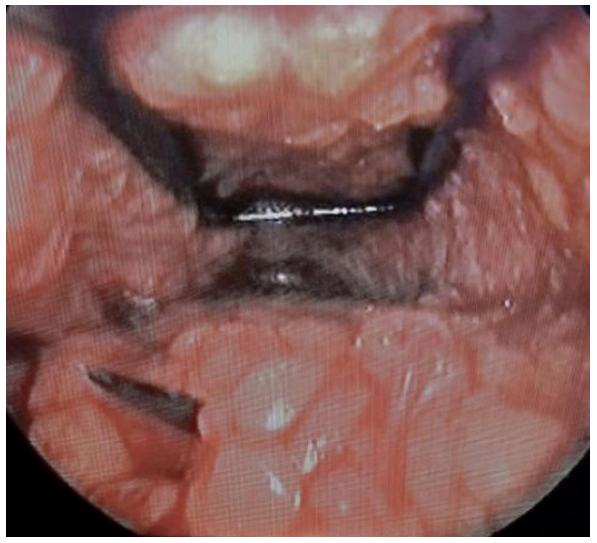

Figure 4: Superficial musculoaponeurosis system suture and imbrication under endoscopic control with the authors' technique

The results are achieved primarily through the correct repositioning of the midface, the appropriate redraping and vector rotation and anchorage of skin flap, all of which are then well supported by SMAS imbrication.

Usage of an endoscope is mandatory. The author advocates video recording of the procedure potentially for accurate documentation in case review is necessary in future. Precise haemostasis is better with endoscopic visualization.

The SMAS must be imbricated to provide stability similar to that of an open procedure [Figure 4]. This is challenging due to the long distance between the incision and imbrication sites. Furthermore, the poor ergonomics of the instrumentation requiring a keyhold like grip makes suturing more difficult.

\section{High malar SMAS anchorage}

The author advocates that the malar fat pad be viewed not as a fat pad, which implies a flimsy structure, but instead as malar SMAS that has implies a stronger structure. The justification for this nomenclature change is due to the reinforced and sturdy nature of both parts of this fat pad described above. Furthermore, usage of the new term this way is key to understanding the role of the malar SMAS for midface repositioning, special threads, special needles and anchoring points. The malar SMAS can be directly repositioned and anchored high on the zygomatic bone, approximately $2 \mathrm{~cm}$ below the horizontal level of the corner of the eye in the middle third of the zygomatic bone to effect the midface lift. This is what the author terms high malar SMAS anchorage and is the cornerstone-working step of the TEM.

The author uses permanent sutures for this purpose and specifically obtains informed patient consent for this purpose. Braided, non-resorbable silicone-coated 


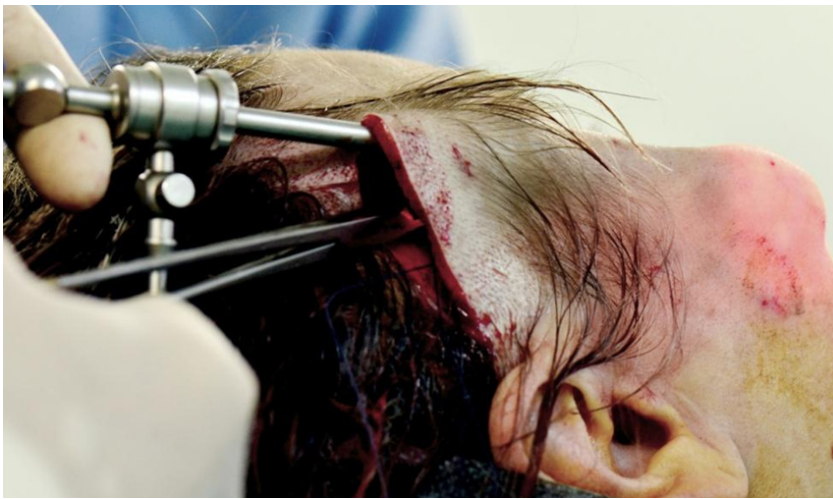

Figure 5: Endoscopic suture technique of the author

polyester threads (Astralen-R Assut Europe, Italy) are used with good results and no reactions. The thread strength for the malar SMAS should be at least USP 0-0 or USP 1-0 to avoid soft tissue cut-through after anchoring or imbrication. The anchoring suture is placed into the stable fascia or periosteum of the zygomatic bone at the high level described above. In selected cases, additional subperiosteal mobilization of the midface through the trans-oral approach is an option to achieve tension-free midface repositioning and anchorage.

\section{The author's method of midface fixation}

It is difficult to work with thick sutures and large needles of $24 \mathrm{~mm}$ or $36 \mathrm{~mm}$ length through an endoscopic tunnel. The author devised a solution to this with a technique as follows: The first stitch is placed through the skin, from the outside into the inside of the endoscopic cavity [Figure 5]. This stitch also catches the SMAS under endoscopic visualization and control. The suturing continues within the endoscopic cavity, the patency of which is maintained with the endoscopic dissector. The suture thread is then completely pulled into the endoscopic cavity and knotted instrumentally.

\section{Direct internal flap anchorage}

The redraping and stable attachment of the skin flap onto the temple is very important for lift stability and longevity. It is insufficient to rely on SMAS imbrications and the high malar SMAS lift for this. Internal flap fixation is a necessary adjunct to impart this stable attachment and has the added benefit of relieving skin tension at the wound edges [Figure 6]. This is done by repositioning, rotating and internally anchoring the skin flap.

It will be taken out by placing USP 2-0 or USP 3-0 polyglactin sutures onto the internal surface of the skin flap in two points as follows: The first skin anchoring

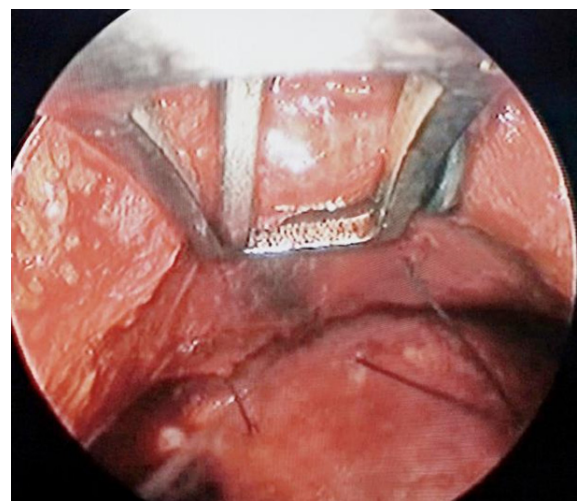

Figure 6: Internal anchoring suture of the skin flap into the inside of the flap

point is about $1 \mathrm{~cm}$ below the horizontal level of the corner of the eye which is anchored onto in the middle third of the zygomatic bone. The second internal skin anchorage connect the skin with the the lateral third of the zygoma or the temporal fascia at the projection of the hairline [Figure 6]. Both of the internal skin flap sutures involve the Caper's fascia of the skin but none of them should cause a visible skin dimple after knotting them. However small dimples can be good managed by subcision and/or fat or PRP filling, which are proposed for volume reconstruction and deflation correction in the postoperative period in any case of facial aging.

As a result, the skin obtains the necessary redraping with long-term internal fixation and skin tension at the temporal wound is alleviated. The skin-to-malar, zygomatic or temporal fascial anchors located $4-5 \mathrm{~cm}$ caudal to the wound edge, have instead taken up this tension. These internal skin flap anchors shorten the distance between the sagging and fixation points.

By a conventional facelift, the skin is pulled at the level of the wound edges, far from the saging skin parts in the midface, which should be elevated. A conventional facelift is therefore not effective for the redraping, reposition and fixation of the midface skin. This disadvantage of the average facelift technique is compensated by internal skin fixation of the TEM lift. A further advantage of the internal skin anchorage is to prevent pulling of the hair bearing skin, to prevent excising too much from it and to prevent sliding back the hairline too much. The goal of any facelift is to elevate the sagging soft tissues. If only skin sutures alone are used to hold the sagging tissues, the skin will stretch out over a short time, resulting in loss of lifting efficiency and longevity. Resorbable threads with strength of USP 3-0 are used for this purpose and should not cause flap ischemia. If the anchoring sutures are well laid out, the yield of the skin surplus is approximately $1-2 \mathrm{~cm}$ in the temporal wound [Figure 7]. 


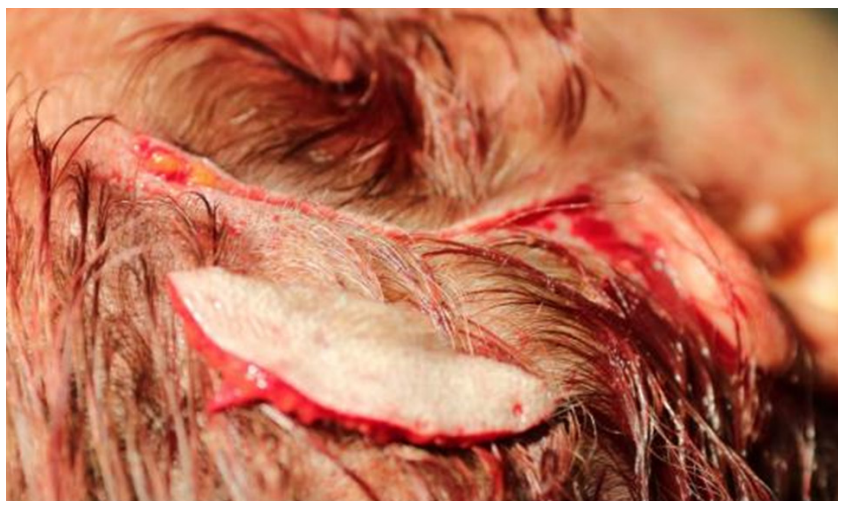

Figure 7: The skin adapts itself without tension after removal of the excess skin

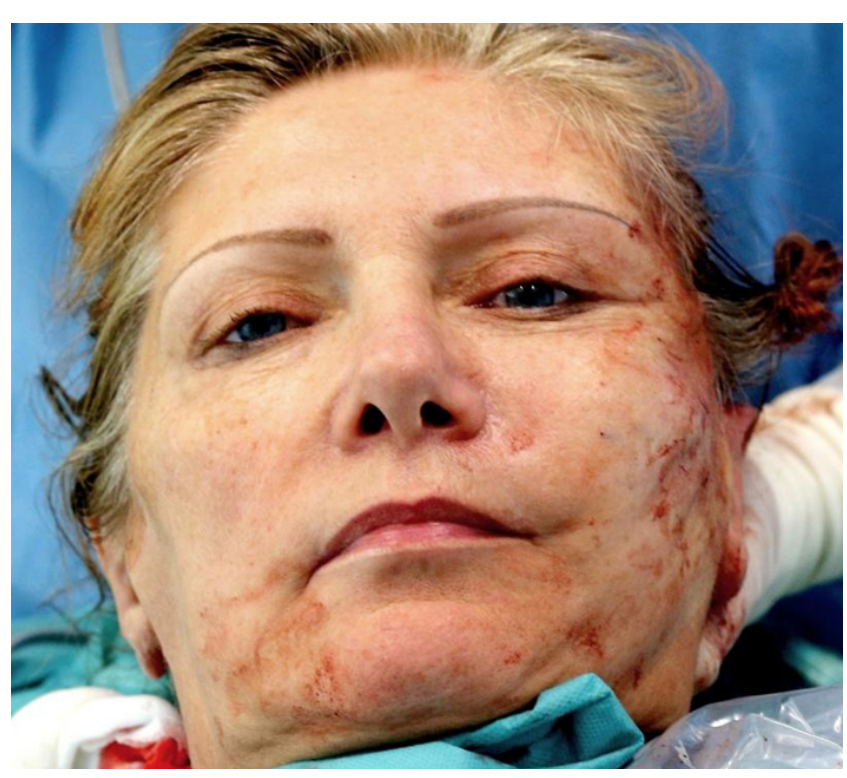

Figure 8: Demonstrating the efficiency of temporal endoscopic midface by an intraoperative shot after completing of the left side. Fully reposition and anchoring of flat malar tissues and the sagging jowls on the left side. Note that the operation takes place under full local anesthesia, an anaesthesiologist is not present during our facelift. The patient can feel and can also give a response in cases, if the operateur works nearby to the nerves. The patient can help the surgeon to prevent nerve injuries

After excising the excess skin, the skin adapts itself well and can be closed tension-free with USP 5-0 interrupted sutures.

\section{Anaesthesia for TEM lift}

Facial procedures are performed in the authors practice with a combination of sedation and local anaesthesia [Figure 8]. Approximately 100-200 mL of Klein's tumescence solution is used for local anaesthesia. The solution is made up with the following components: $500 \mathrm{~mL} 0.9 \% \mathrm{NaCl}, 50 \mathrm{~mL}$ of $2 \%$ Lidocaine, $1 \mathrm{~mL}$ of $1 \mathrm{mg} / \mathrm{mL}$ epinephrine 4 from Adrenalin ${ }^{\circledR}$. A dose of $5 \mathrm{mg}$ Midazolam is given as premedication. Patients also receive 3-5 mg of Piritramide intravenously before the local anaesthesia infiltration is administered. We have not observed any pain or other complications during the last 15 years using this simple and effective method, which can be applied to more extensive open face and midface lifts.

\section{Bandaging}

We apply elastic bandaging to the head for the first two postoperative days. Then we use kinesio tapes for the stabilization of the operation results during the first week, similar to that used by athletes for sport injuries. These tapes promote healing by preserving the lift as well as improving the dynamic lymphatic drainage. This thereby contributes to a quicker reduction in swelling. Conventional dressings may be omitted after the second day.

\section{RESULTS}

The lifting effect of the temporal endoscopic midface lift extends from the eyebrows to the midface, and affects also the jawline and neck [Figures 9-13]. The operation is relatively quick and takes two and a half to three hours in the hands of an experienced endoscopic surgeon. The postoperative results appear very natural and do not suffer from an "operated" look. The author attributes this to lifting using the correct vector against the force of gravity. The healing period takes approximately 14 days, and largely dependent on the variable resolution of oedema and swollen eyes. We stress that overcorrection is very important, which requires a calculated distortion. This distortion that is similar in appearance to "almond eyes" or "cat eyes" may persist for two to four weeks prior to resolution.

There are no facelift stigmas or conspicuous scars on the face. The only scar is located within the temporal
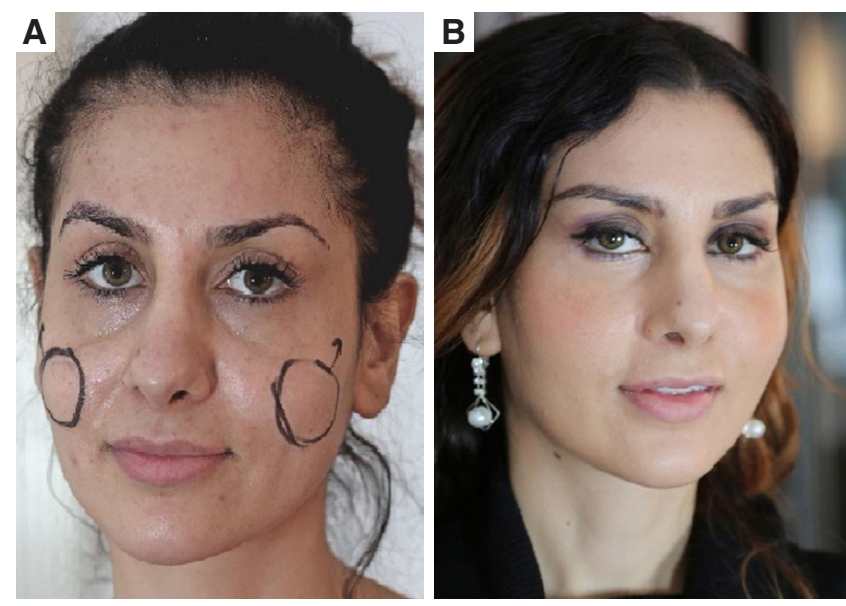

Figure 9: Before (A) and 6 months after temporal endoscopic midface (B). More pleasant looking after endoscopic midface reposition by diminishing the suborbicular hollowing 

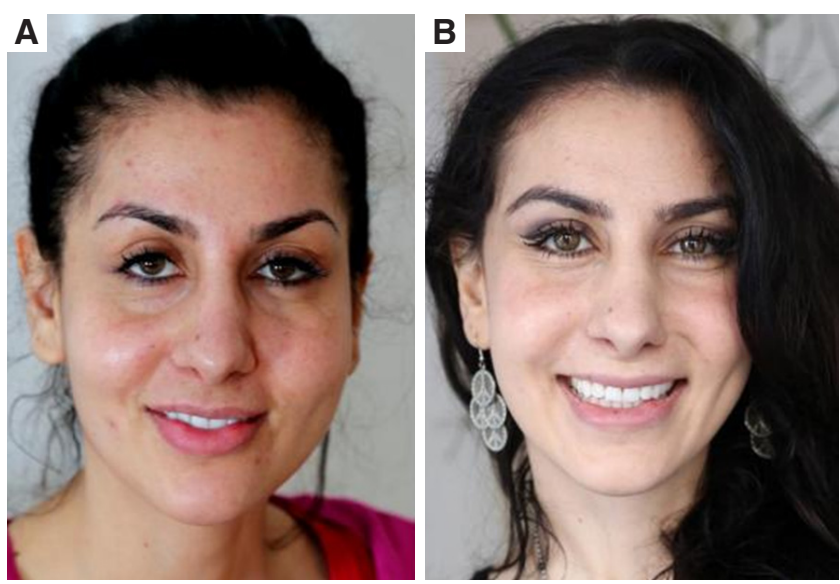

Figure 10: Before (A) and after (B) temporal endoscopic midface. Note also the juvenile $\mathrm{V}$-form of the jaws 6 months after the scar free face lifting. Note the psychological status of the patient and her evident feeling of happiness
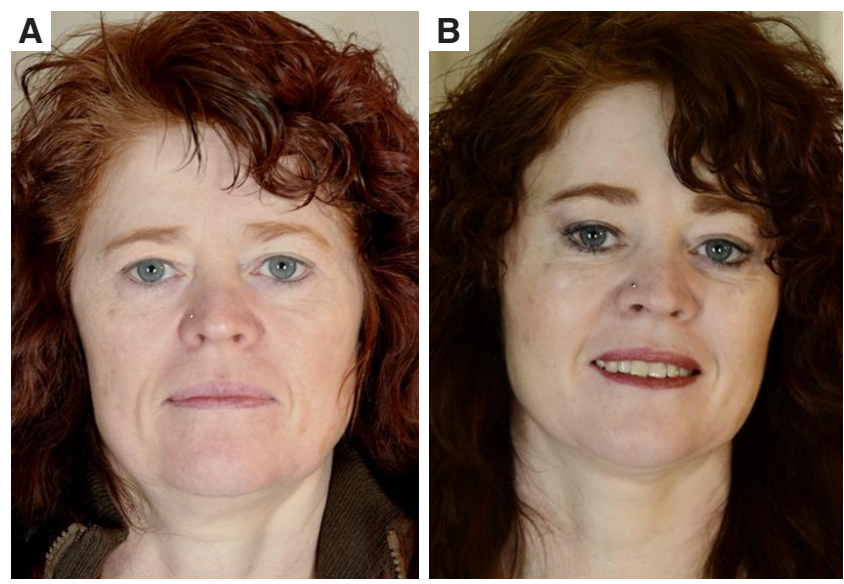

Figure 12: Before (A) and after (B) temporal endoscopic midface. Visible rejuvenation and improving also of the psychological state of the 48-year-old patient

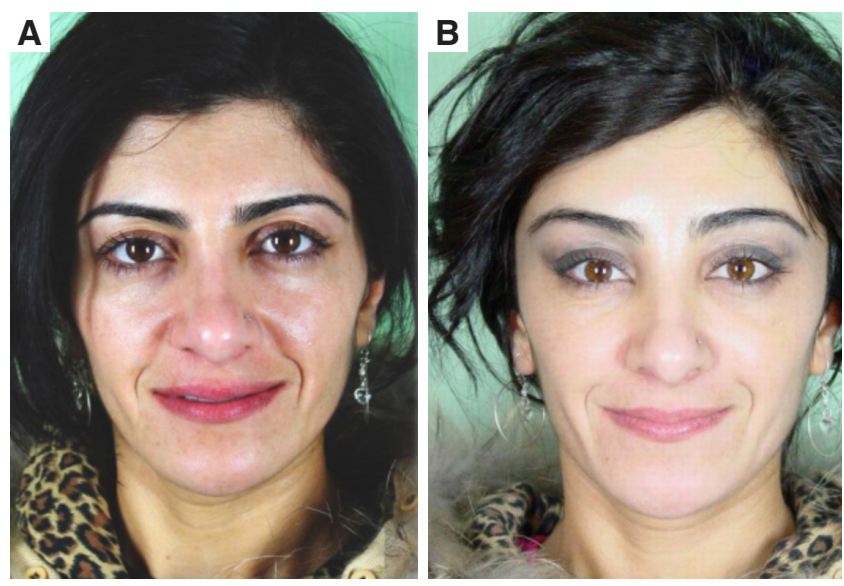

Figure 14: Before $(A)$ and 3 months after $(B)$ temporal endoscopic midface (TEM) lift by a 30 -year-old patient. Note the tired look before and the pleasant refreshing effect after TEM lift with improved brow position, with correction of the suborbicular hollowing. Improving of the nasolabial folds and the centrofacial depletion by correct reposition of the mid-facial structures
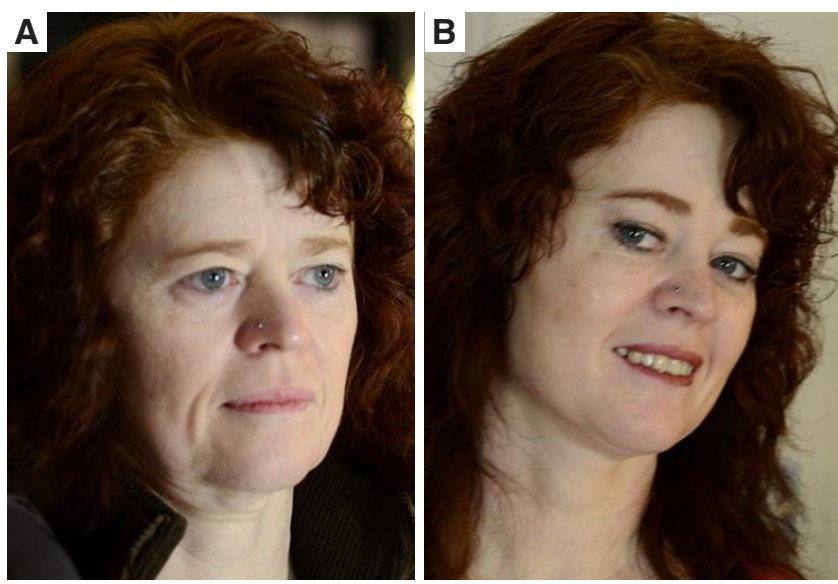

Figure 11: Before (A) and after (B) temporal endoscopic midface. A 48-year-old patient, note the improved contours of the malar and peri orbicular regions and of the jawline resulting much more pleasing face
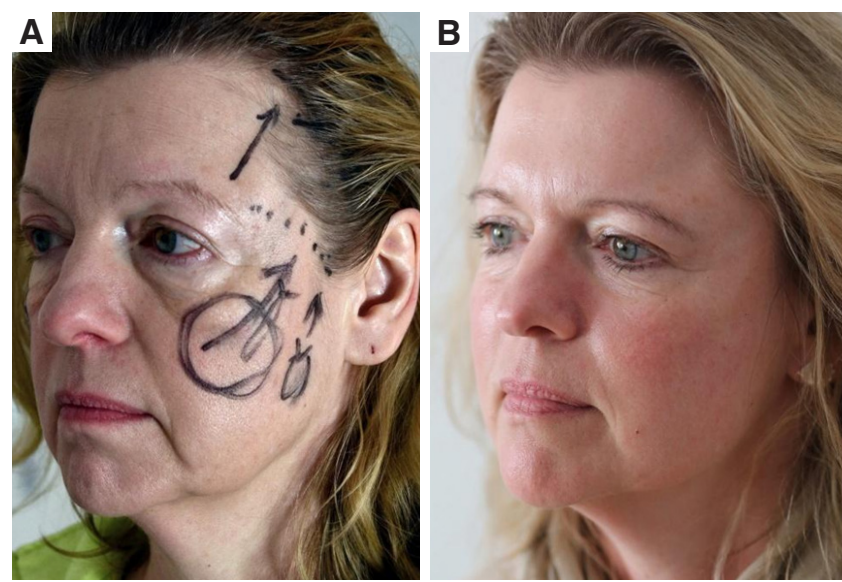

Figure 13: Before (A) and 6 months after $(B)$ the procedure by a 55 -year-old patient. Note the improving of the suborbicular region by temporal endoscopic midface. No blepharoplasty was performed

hairline. It is therefore barely visible and can be well covered by the adjacent hair. Broad scarring with scar alopecia can be avoided with proper dissection and suture techniques. Due to the lack of facial scars, TEM is the method of choice for scarless facial rejuvenation. TEM is appropriate when no large jowls or skin excesses in the neck are present and is suited especially for young women and men who frequently lack such excesses [Figures 13-15].

\section{DISCUSSION}

Aging process is a combination of ptosis, deflation and wrinkling. A facelift corrects the ptosis of the lateral facial parts and of the jaws and neck, but not really works against centrofacial aging. As it described before the mid facial structures are not sufficient repositioned by a conventional facelift - they are too far from the large lateral acces and the scar means a 

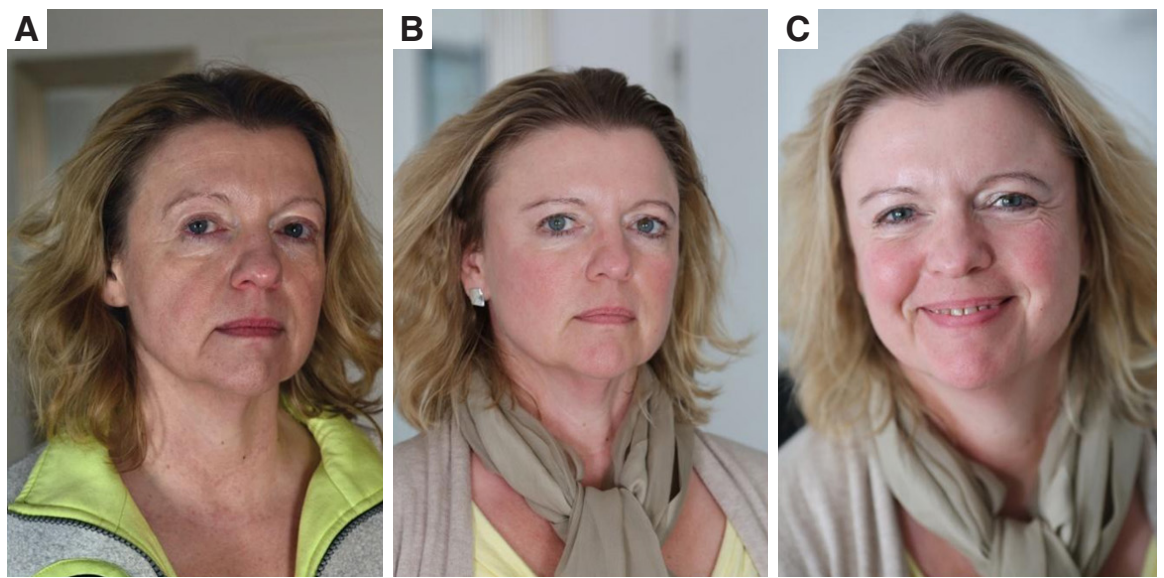

Figure 15: Before $(A)$ and 6 months after $(B$ and $C)$ temporal endoscopic midface. Note the freshness of the whole face, the diminishing of the nasolabial folds, more malar fullness, pleasant periorbital of the 55-year-old patient. A proper repositioned midface with more malar fullness, diminishing of jowling and of the nasojugal folds 6 months after temporal endoscopic midface by a middle aged woman with happiness and satisfaction

stigma forever. By the MACS lift ${ }^{[10]}$ the lateral face and the jawline can be well corrected, howewer on the cost of a pretrichal and praearicular scar. The so-called "third suture" of the midface ${ }^{[10]}$ is also an additive element by the necessity of midface restoration even by young individuals. There are many subperiosteal ways of accesses to the midface to reposition and restore it efficient. All of them lack to generate a unity of the SMAS and also of the skin layer lifting each of them in one block, in one common layer.

In case of a temporal subperiosteal lifting, the fully reposition is partly blocked by the anatomical adherence of the SMAS and the facial nerve onto the zygomatic bone. The anchorage means only a suspensions lift, which does not allow the sunken part to merge with the part where the sunken part is anchored. It is then only the question of believing or not believing, whether any part of the body can be holded on a cord or "cable" with longevity.

The most effectively vertical midface reposition with direct anchorage is practiced by Botti and Ceravolo, ${ }^{[1]}$ which was also adopted by the author. Howewer, a transblepharoplasty vertical, subperiosteal midface lift supposes an extended lower lid correction with canthopexy, though has many risks of a lower lid correction such as ectropion, scleral show, conjunctivitis, chemosis, lagophthalmos, assymetry, negative tilt, rounding of the eyes. The procedure needs drilling for bony tunnels, then also skin excision periorbital or temporal, that means three procedures in the same time: extended lower lid correction plus midface subperiosteal lift plus temporal lift. If a consent is correctly made, then such a multiplex combinative procedure will be mostly refused by a young patient and also very rarely can be indicated, than real ptosis and sagging are not common by young patients. Rather a centrofacial depletion is the case by such individuals, which is best treated by sharp needle intradermal fat or nano fat or micro fat injections in the hands of Verpaele et al. ${ }^{[10]}$ and by the author.

The rejuvenation effect of the TEM lift is directed on the centro- and mid- facial sagging tissues and therefore adresses the roots of deflation and flattening of the midface by young patients. The reposition of the malar fat pad in combination with tightening both of the SMAS and of the skin make a natural volume restoration malar and submalar [Figure 8] and affect also the jawline [Figures 9-12]. The jaws get the natural and youthful $\mathrm{V}$ - form instead of quadratic form of jawling after the TEM Lift.

So the TEM incorporates the advantages as follows: rejuvenation in the problematic centrofacial region, volume restoration by reposition, affect also the lateral jawline and thus without any facial stigma.

Candidates of the TEM lift are prevented from possible complications of a lower lid operation and needs for the centrofacial reposition only one procedure and not three as like by transblepharoplasty subperiosteal midface lift. For young patients without severe sagging and deflation of the midface and/or without severe jawling and neck problem this scar savin procedure seems to be the best choice of by centrofacial rejuvenation by a restorative way.

An additive volume restoration should be however an adjuctive part of any rejuvenation of the face, than both the ptosis and the volume loss should be corrected in all parts of the face according to our conviction. 
Older patients with significant skin excess of the jaw and neck are better suited for conventional lower facelift surgeries, intermediate cases can get a restricted pretrichial neck lift added to the scar sparing TEM lift by the author.

\section{Financial support and sponsorship \\ None.}

\section{Conflicts of interest}

There are no conflicts of interest.

\section{Patient consent}

All operations and photographs were performed after the patients' written consent.

\section{Ethics approval}

Ethics approval was given by all demonstrated patients for the presentation.

\section{REFERENCES}

1. Botti G, Ceravolo MP. Midface and Neck Aesthetic Plastic Surgery. Volume 1: Midface. 1st ed. Florence: Springer; 2012. p. 560.
2. LaFerriere KA, Kilpatrick JK. Transblepharoplasty: subperiostea approach to rejuvenation of the aging midface. Facial Plast Surg 2003;19:157-70.

3. Mofid MM. A novel technique for repositioning lower eyelid fat via the trans-oral approach in association with midface lift. Aesthetic Plast Surg 2011;35:563-8.

4. Quatela VC, Antunes MB. Trans-temporal midface lifting to blend the lower eyelid-cheek junction. Clin Plast Surg 2015;42:103-14.

5. Ramirez OM. Three-dimensional endoscopic midface enhancement: a personal quest for the ideal cheek rejuvenation. Plast Reconstr Surg 2002;109:329-40; discussion 341-9.

6. Ransom ER, Stong BC, Jacono AA. Persistent improvement in lower eyelid-cheek contour after a trans-temporal midface lift. Aesth Plast Surg 2012;36:1277-82.

7. de la Fuente A, Hönig JF. Video-assisted endoscopic trans-temporal multilayer upper midface lift (MUM-Lift). J Craniofac Surg 2005;16:267-76.

8. Viksraitis S, Astrauskas T, Karbonskiene A, Budnikas G. Endoscopic aesthetic facial surgery: technique and results. Medicina (Kaunas) 2004;40:149-55. (in English, Lithuanian)

9. Williams EF 3rd, Vargas H, Dahiya R, Hove CR, Rodgers BJ, Lam SM. Midfacial rejuvenation via a minimal-incision brow-lift approach: critical evaluation of a 5-year experience. Arch Facial Plast Surg 2003;5:470-8.

10. Verpaele A, Tonnard P, Gaia S, Guerao FP, Pirayesh A. The third suture in MACS-lifting: making midface-lifting simple and safe. $J$ Plast Reconstr Aesthet Surg 2007;60:1287-95.

11. Haffner T. Das temporal-endoskopische Mittelgesichtslift Gesichtslifting ohne Gesichtsnarben. Jf Ästhet Chirurgie 2014;7:98-105. (in German) 